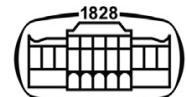

AKADÉMIAI KIADÓ

Journal of Behavioral Addictions

9 (2020) 4, 1002-1010

DOI:

$10.1556 / 2006.2020 .00104$

(c) 2020 The Author(s)

\section{FULL-LENGTH REPORT}

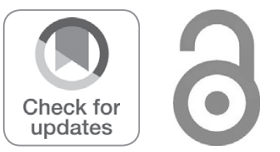

\title{
Mediating effects of rumination and bedtime procrastination on the relationship between Internet addiction and poor sleep quality
}

\author{
ZHIQI YOU ${ }^{1}$, WEIJIE MEI ${ }^{1}$, NA YE ${ }^{2}$, LU ZHANG ${ }^{1^{*}} \oplus$ and \\ FRANK ANDRASIK ${ }^{3^{*}}$
}

\author{
${ }^{1}$ Department of Social Work, Huazhong Agricultural University, Wuhan, 430070, China \\ ${ }^{2}$ Department of Psychology, Wuhan Sports University, Wuhan 430079, China \\ ${ }^{3}$ Department of Psychology, University of Memphis, Memphis, TN 38152, USA
}

Received: July 05, 2020 • Revised manuscript received: November 04, 2020 • Accepted: December 06, 2020

Published online: December 31, 2020

\begin{abstract}
Background and aims: Numerous studies have shown that people who have Internet addiction (IA) are more likely to experience poor sleep quality than people who do not. However, few studies have explored mechanisms underlying the relation between IA and poor sleep quality. As a first attempt to address this knowledge gap, a cross-sectional design was applied, and structural equation modeling was used to explore the direct relationship between IA and poor sleep quality, as well as the potential mediating roles of rumination and bedtime procrastination. Methods: A convenience sample, consisting of 1,104 Chinese University students (696 females or 63\%), completed an online survey that included the following measures: Young's 8-item Internet Addiction Diagnosis Questionnaire, the Pittsburgh Sleep Quality Index, the Ruminative Responses Scale, and the Bedtime Procrastination Scale. Results: While the direct path between IA and poor sleep quality was not found to be significant, rumination and bedtime procrastination were each shown to separately mediate the predictive effect of IA on poor sleep quality. However, the greatest level of support was found for the sequential mediating effects of rumination and bedtime procrastination between IA and poor sleep quality. Conclusion: While rumination and bedtime procrastination were both shown to be important independent mediators for the relation between IA and poor sleep quality, their combined effect was as great as either alone.
\end{abstract}

\section{KEYWORDS}

Internet addiction, poor sleep quality, rumination, bedtime procrastination

\section{INTRODUCTION}

Internet addiction (IA), also known as problematic Internet use, refers to the inability of individuals to control their use of the Internet, leading to panic and dysfunction in their lives (Vadher et al., 2019). Reports on the prevalence of IA among university students have varied considerably, ranging from approximately $4 \%$ in the USA (Christakis, Moreno, Jelenchick, Myaing, \& Zhou, 2011), 16.8\% in Italy (Younes et al., 2016), to higher rates from 17 to $26.8 \%$ in China (Shek \& Yu, 2016). IA, consequently, has become one of the main factors affecting the physical and mental health of college students (e.g., Bener et al., 2019; Chen et al., 2020). One of the most common detrimental effects of IA is disruption of sleep quality (Wang, 2018), such as

"Corresponding authors. E-mail: zhanglu@mail.hzau.edu.cn; fndrasik@memphis.edu disturbance of one's circadian rhythm and development of a formal sleep disorder (Feng, Zeng, Sun, \& Kuroki, 2014). The relation between IA and sleep problems has also been confirmed in meta-analytic and systematic reviews (e.g., Alimoradi et al., 2019; Lam, 2014).

The predictive effect of IA on subjective sleep quality (measured by the Pittsburgh Sleep Quality Index) has been explored in several large sample cross-sectional studies among college students (e.g., Bener et al., 2019; Jahan et al., 2019; Wang et al., 2020) and adolescents 
(e.g., Vadher et al., 2019; Çelebioğlu, Aytekin Özdemir, Küçükoğlu, \& Ayran, 2020). For example, the cross-sectional study by Wang et al. (2020) found that Chinese college students with IA had a 3.25 times greater odds ratio of experiencing poor subjective sleep quality. However, most previous studies have been designed chiefly to examine for the presence of a stable relationship between IA and sleep quality (e.g., Vadher et al., 2019; Zhang et al., 2017). To our knowledge, few of the published studies have thus far tried to explore mechanisms potentially mediating the relationship between IA and poor sleep quality. Therefore, this study was conducted to investigate the mediating effects of rumination and bedtime procrastination on the relationship between IA and poor sleep quality, which are discussed more fully below.

\section{The mediating role of rumination}

Rumination refers to the tendency to repeatedly and passively think about the negative situations, symptoms, causes and potential consequences of failing to achieve desired goals (Nolen-Hoeksema, 1991). It is a way of thinking that repeatedly focuses on one's own negative emotions and corresponding events (Nolen-Hoeksema \& Morrow, 1991). Studies have shown that ruminative thinking has a significant impact on sleep quality (Liu et al., 2017), with individuals evidencing the highest levels of rumination reporting the most serious problems with sleep quality (Butz \& Stahlberg, 2018). The cognitive model of insomnia (Espie, 2007) points out how pre-sleep sleepinterfering cognitive processing can markedly increase sleep problems. Rumination, or automatic thinking, is an important source of pre-sleep cognitive arousal that makes it increasingly difficult to fall asleep quickly and/or maintain a sound level of restorative sleep (Guastella \& Moulds, 2007).

A cognitive-behavioral model of IA suggests rumination is an important cognitive characteristic of individuals with this condition (Davis, 2001). This model espouses that individuals with IA are unable to prevent negative information from entering working memory and, once retained in working memory, these negative thoughts reoccur repeatedly (Joormann \& Quinn, 2014). Previous research has shown that IA can adversely impact personal offline activities, such as decreasing academic performance and impairing interpersonal relationships. These untoward effects will inevitably increase one's stress burden (Kuss \& Griffiths, 2011), which typically induces and/or exacerbates ruminative thinking (Nolen-Hoeksema, 1991). A recent study showed the partial mediating role that rumination can play in the relationship between mobile phone addiction and poor sleep quality in adolescents (Liu et al., 2017), with these findings used in part to inform our initial hypothesis: Rumination mediates the relationship between IA and poor sleep quality (Hypothesis 1 or $\mathrm{H} 1$ ).

\section{The mediating role of bedtime procrastination}

Bedtime procrastination is defined as failing to go to bed at the intended time absent any external circumstances that prevent a person from doing so (Kroese, Evers, Adriaanse, \& de Ridder, 2016). The procrastination-health model suggests that procrastination is an important risk factor for poor sleep quality (Sirois, 2007), a viewpoint supported by various empirical studies (e.g., Przepiórka, Błachnio, \& Siu, 2019). Researchers have shown that going to bed later than intended due to procrastination significantly predicts poor sleep quality (Kadzikowska-Wrzosek, 2018; Kroese, Evers, Adriaanse, \& de Ridder, 2016), with bedtime procrastination surfacing as a key factor underlying sleep deprivation (Exelmans \& Van den Bulck, 2016; Kroese et al., 2016; Nauts, Kamphorst, Stut, De Ridder, \& Anderson, 2018).

The Temporal Motivation Theory (TMT; Steel \& König, 2006), when applied to procrastination, suggests that people tend to postpone tasks when they view the outcome value of the task is low, and they are not likely to receive either rewards or punishments immediately. People with IA have higher levels of psychological craving for Internet use (Niu et al., 2016), which, in turn, serve to reduce their motivation go to bed at a reasonable time as the health benefits derived from sleeping accrue at a later point in time. This makes them, according to the theory, more inclined to overuse the Internet due to its immediate enjoyment, leading to bedtime procrastination. The Displacement Hypothesis of the Internet (Nie \& Hillygus, 2002) further accounts for the relationship between overuse of the Internet, bedtime procrastination and poor sleep quality. As time is constant for everyone, the greater the amount of time spent online, the less time a person has to spend on activities during the remainder of the day, such as sleeping and engaging in physical activity. Individuals with IA continually delay disengaging from the Internet, leading to bedtime procrastination which decreases time available for sleep, with resultant poor sleep quality (Jahan et al., 2019).

Various studies have similarly shown that excessive time spent on the Internet while in the bedroom and/or in bed not only delays the time to sleep onset, but also contributes to unhealthy sleep habits (such as reading news or playing games on one's mobile phone) that in turn reduce sleep quality (Brunborg et al., 2011; Cain, \& Gradisar, 2010; Suganuma et al., 2007). These unhealthy habits can disrupt the circadian sleep pattern (e.g., sleepless, decreased sleep duration during the night-time; Chen \& Gau, 2016), which in turn contributes to poor sleep quality (Wang, Chen, Yang, \& Lin, 2019). Bedtime procrastination, therefore, may also be an important mediating variable in the relationship between IA and poor sleep quality. Based on the accumulated findings reported here, we hypothesize $(\mathrm{H} 2)$ that bedtime procrastination also mediates the effect of IA on poor sleep quality.

\section{Serial mediating effect of rumination and bedtime procrastination}

Stainton, Lay, and Flett (2000) argued that rumination is an important factor affecting procrastination behavior, which has been supported by many investigations (e.g., Constantin, English, \& Mazmanian, 2018; Gort, Marcusson-Clavertz, \& 
Kuehner, 2020; Rebetez, Rochat, Barsics, \& Van der, 2018). Studies have also found that rumination can significantly predict procrastination; as when levels of rumination increase so do procrastinating behaviors (Flett, Haghbin, \& Pychyl, 2016). Bedtime procrastination, a specific form of procrastination, may also be affected by rumination. The association between rumination and bedtime procrastination may be viewed from several perspectives. For example, ruminative behavior has been shown to evoke increased negative affect (Lai, Han, \& Yang, 2009), and one way of coping with such negative affect is to delay time sleeping (Sirois, Nauts, \& Molnar, 2019). High levels of rumination can also evoke a sense of time loss (Wood, Griffiths, \& Parke, 2007), which is manifested by people losing track of time and thereby gradually increasing bedtime procrastination. Thus, in this study, we hypothesize (H3) that IA may indirectly affect sleep quality through the sequential mediating effects of rumination and bedtime procrastination.

Consideration of the above led us to conduct this study to explore the mediating effects of rumination and bedtime procrastination in the relationship between IA and poor sleep quality in order to further clarify the mechanisms underlying the predictive power of IA on sleep quality. Tests of our 3 specific hypotheses were formulated to provide further theoretical insights that may lead to improved ways to address sleep problems that youth experience due to IA.

\section{METHODS}

\section{Participations and procedure}

An online survey was used to collect data in the month of December 2017. First, we selected the largest, professional and most frequently used (e.g., Lu, Liu, Liao, \& Wang, 2020; Peng \& Xie, 2016) online survey platform in China (Wenjuanxing; www.wjx) for this purpose. Second, 19 research assistants (undergraduate students) were trained on how to administer the survey. Each research assistant was then sent an advertisement containing the introduction to the survey and a link to the on-line survey, produced by the Wenjuanxing platform, to connect with the WeChat group or QQ group of their classes or their friends' classes. Consequently, it was not possible to calculate the number of potential respondents and completion rates (Callegaro \& DiSogra, 2008). The purpose of the survey was explained and students were informed that their participation was completely voluntary and that any information they provided would be anonymous in order to protect their privacy rights. Prior to volunteering and clicking on the link to the questionnaire, respondents were notified that they were free to withdraw from the survey at any time without any penalty. Survey data collection was designed so that a participant could submit only one response using the same IP address.

A total of 1,367 respondents completed and submitted the survey. Recognition of the fact that very short response times typically indicate low data quality in online surveys (Greszki, Meyer, \& Schoen, 2015), 169 cases were subsequently eliminated, using the approach recommended by Greszki et al. Omitted were those individuals whose completion times were less than 60 percent of the median ( $60 \%$ median of response time $=285$ seconds). An additional 94 cases that contained response errors were also omitted (e.g., answering in an identical manner to each question, such as responding " 2 " to all items; failing to fully complete all survey items; marking multiple responses for a given question, etc.). The resultant sample consisted of 1,104 valid questionnaires (696 or $63 \%$ females and 408 or $37 \%$ males), with respondents ranging in age from 17 to 31 years, with a mean age of $20.2(\mathrm{SD}=1.43)$ years.

\section{Measures}

Internet Addiction Diagnosis Questionnaire. Young's (1998) 8-item Internet Addiction Diagnosis Questionnaire (IAD), designed as one of the first to investigate the presence of addiction to the Internet, was subsequently revised and translated into Chinese (Cui \& Wang, 2003) and validated in various studies and a meta-analysis (Kuang, Cao, \& Dai, 2011; Li et al., 2012). We used the resultant Chinese version to quantify the degree of impairment in academic, social, and occupational domains by misuse or overuse of the Internet (e.g., preoccupation, need for increased amounts of time to be satisfied, repeated unsuccessful attempts to control use, etc.). In order to increase the variance for the obtained scores, subsequent versions of the scale have replaced the original dichotomous yes-no response format with various Likert scale formats, ranging in point values (e.g., Andreassen et al., 2013; Bai \& Fan, 2005). The Chinese translated and validated version of the IAD we used incorporated a 6-point Likert scale, where $1=$ "extremely disagree" to $6=$ "extremely agree". The average of the individual items was used to reflect the extent to which people engage in IA behavior. Individuals were classified as having IA if they chose a score of 5 or more ("agree" or "extremely agree") for at least 5 of the total 8 items. In this study, the internal consistency coefficient of the scale was 0.91 .

Pittsburgh Sleep Quality Index. The Pittsburgh Sleep Quality Index (PSQI) was adapted from the original version developed by Buysse, Reynolds, Monk, Berman, and Kupfer (1989), to evaluate sleep quality. In completing this measure, respondents are asked to rate each of 19 items assessing various aspects of sleep, based on recall over the past month. The 19 items are grouped into 7 components, each weighted equally on a scale ranging from 0 to 3 . Adding the 7 separate domain scores yields a global measure of sleep quality that ranges from 0 to 21 , with higher scores representing poorer sleep quality. This questionnaire was translated and validated among the Chinese population (Liu, Tang, \& Hu, 1996), with scores $>8$ indicative of poor sleep quality. The alpha coefficient for the questionnaire as administered in this study was 0.80 .

Ruminative Responses Scale. The Ruminative Responses Scale (RRS), a subscale of the more extended Response Styles Questionnaire, developed by Nolen-Hoeksema and Morrow 
(1991), was used to capture 2 general aspects of rumination - "brooding" and "reflective pondering" (Treynor, Gonzalez, \& Nolen-Hoeksema, 2003). This scale consists of 22 items assessing responses focused on one's self (e.g., "Why do I have problems that other people don't have?"), specific symptoms (e.g., "Think about your feelings of fatigue and achiness."), and causes or consequences of being in a depressed mood (e.g., "I won't be able to do my job if I don't snap out of this."). Each item is rated on 4-point scale, where 1 = "almost never" to $4=$ "almost always." Item mean scores were used in analyses to facilitate interpretation of the 1-4 response scale. This scale was translated and validated among a Chinese population (Han \& Yang, 2009), with the alpha coefficient for the scale as administered in this study being 0.94 (slightly higher than the internal consistency value reported for the original version, 0.82).

Bedtime Procrastination Scale. The Bedtime Procrastination Scale (BPS; Kroese, de Ridder, Evers, \& Adriaanse, 2014) is designed to measure the extent to which a person goes to bed later than intended, absent any external circumstances to delay. The BPS contains 9 statements (e.g., "I go to bed later than I had intended," "Often I am still doing other things when it is time to go to bed"), with each being rated on a 5point scale, where 1 = "almost never" to 5 = "almost always" (with some items reverse scored to minimize response biases). Higher total scores reflect increased procrastination. For our purposes, this scale was translated into Chinese, as follows: The directions and individual items were first translated and then back translated by a university English teacher and three Professors who have doctoral degrees and teach psychology in a university. The resultant Chinese version was subsequently submitted to a confirmatory factor analysis following the suggestion of Straub (1989), which revealed our Chinese version of the BPS possessed good construct validity (Chi-square $=142.72, \mathrm{df}=22, P<0.01, \mathrm{RMSEA}=0.07$, CFI $=0.99$, GFI $=0.97$ ). The alpha coefficient for our translated scale was 0.88 . We opted to use the mean response for all items when analyzing BPS scores.

\section{Statistical analysis}

Structural equation modeling (SEM) and maximum likelihood functions, conducted with Mplus 7.0 for Windows, were used to model variable relationships, which are considered particularly suitable when analyzing large crosssectional data sets (Kline, 2015). Ten individuals, whose responses were otherwise complete, neglected to report their age, leading us to impute their age. Given the reported range of ages was quite small, we used the mean age for the entire sample for each of these 10 individuals. For our primary analyses, we first computed the univariate and multivariate skewness and kurtosis for a multivariate normal distribution test. Then, we repeatedly drew a number of random samples (a total of 5,000 , each with a sample size of 1,104 ) based on the original sample, which yielded a robust standard error of the parameter estimation and confidence intervals (as suggested by Tofighi, 2020; Tofighi \& Kelley, 2020). In this approach, confidence intervals that do not contain zero indicate the presence of statistical significance.

\section{Ethics}

The research was approved by the Institutional Review Board at Huazhong Agricultural University (HZAU), and conforms with the APA ethical standards for conducting research investigations, the Declaration of Helsinki, as well as the ethical guidelines for human subject research.

\section{RESULTS}

\section{Descriptive analyses}

Two hundred-eighty (280) college students (25.4\%) were classified as having IA behavior, which is consistent with the previous studies (e.g., Chung, Sum, \& Chan, 2019; Shek \& $\mathrm{Yu}, 2016)$. A near identical percentage $(25.5 \%, n=281)$ were identified as having poor sleep quality, which also accords with the previous studies (e.g., Falavigna et al., 2011). Mean values (along with SDs) and the Pearson productmoment correlation coefficients between all study variables, for all 1,104 participants, are presented in Table 1. All pairwise correlation coefficients were positive and significant, with the lowest percentage of variance accounted for being $9 \%$ and the highest being $21 \%$.

\section{The multiple mediation model}

Figure 1 illustrates the findings from the full structural equation model used to analyze the hypotheses of this study. After controlling for the effects of age and sex, the full model provided a good fit to the data (RMSEA $=0.04$, CFI $=0.98$, TLI $=0.94$, SRMR $=0.05$, Chi-square $=9.52, \mathrm{df}=4, P<$ 0.05 ) and accounted for $32.2 \%$ of the variance in poor sleep

Table 1. Descriptive statistics and correlations among variables

\begin{tabular}{lccrrr}
\hline & $\mathrm{M} \pm \mathrm{SD}$ & 1 & 2 & 3 & \\
\hline 1. Internet addiction & $2.06 \pm 0.51$ & - & & \\
2. Poor sleep quality & $5.93 \pm 2.69$ & $0.34^{* *}$ & - & - \\
3. Rumination & $3.22 \pm 0.77$ & $0.46^{* * * *}$ & $0.39^{* *}$ & $0.30^{* *}$ & - \\
4. Bedtime procrastination & $4.17 \pm 0.84$ & $0.35^{* * *}$ & $0.41^{* *}$ & -0.05 \\
Age & $20.17 \pm 1.43$ & -0.04 & $0.06^{*}$ & -0.05 & 0.00 \\
Sex & - & 0.01 & -0.06 & & $0.06^{*}$ \\
\hline
\end{tabular}

Note: ${ }^{*} P<0.05 ;{ }^{* *} P<0.01$. male $=1$; female $=0$. 


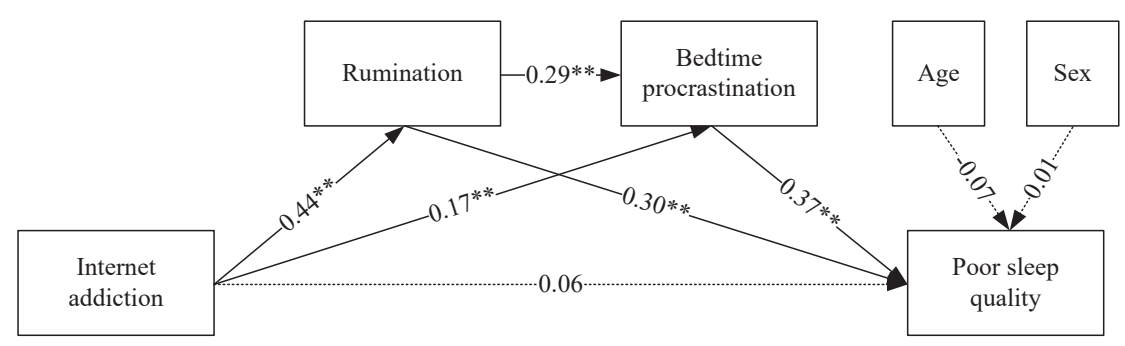

Fig. 1. Pathway of Internet addiction affecting poor sleep quality (all path coefficients were standardized; the dashed line indicates the single nonsignificant path, $\left.{ }^{* *} P<0.01\right)$

quality. All of the coefficients of the paths in the full model were significant $(P<0.01)$ except the direct path between IA and poor sleep quality $(\beta=0.06, P>0.05)$.

To further evaluate the indirect effect of this model, we first tested the univariate and multivariate normality, applying the method developed by Cain, Zhang and Yuan (2017). Skewness (Kurtosis) for poor sleep quality, IA, rumination and bedtime procrastination was $0.59(0.67)$, $0.28(-0.63), 0.63(0.98),-0.30(-0.51)$, respectively. The results of Mardia's multivariate skewness and kurtosis (Mardia, $1970)$ were $1.26(Z=231.83, P<0.01)$ and $27.49(Z=8.36, P$ $<0.01)$, leading us to reject the hypothesis of multivariate normality. Drawing upon the recommendations of Tofighi (2020) and Tofighi and Kelley (2020), we tested our hypothesized model (Fig. 1) with 5,000 bootstrap samples to compute percentile $95 \%$ confidence intervals for the indirect effects. The mediating effect of rumination on the relationship between IA and poor sleep quality was found to be significant, supporting $\mathrm{H} 1$, as was the mediating effect of bedtime procrastination between IA and poor sleep quality, supporting H2. Perhaps most of interest was the significant findings for the sequential mediating effect of rumination and bedtime procrastination on the relationship between IA and poor sleep quality, supporting H3. Thus, all hypotheses were supported, with rumination and bedtime procrastination fully mediating the influence of IA on poor sleep quality (Table 2).

\section{DISCUSSION}

Our results, which showed that IA by itself was not predictive of poor sleep quality, did document the independent mediating effects of both rumination (H1) and bedtime procrastination (H2) between IA and poor sleep quality. Most important, however, was the support found for the sequential mediating effects of rumination and bedtime procrastination $(\mathrm{H} 3)$ between IA and poor sleep quality. These findings substantiate the notion that bedtime procrastination results at least in part by inadequate self-regulation of negative affect (Exelmans \& Van den Bulck, 2018) that arises from heightened levels of rumination. They also conform with the known tendencies of people who have IA to focus on immediate rewards and ignore the long-term negative results in decision-making (Pawlikowski \& Brand, 2011). TMT, advanced by Steel and König (2006), is able to explain the sequential or combined mediating effects as follows: Persons with IA tend to ignore the long-term values of going to bed on time, preferring the immediately pleasurable effects derived from engaging with the Internet, which leads to bedtime procrastination (Exelmans \& Van den Bulck, 2017). When considered in conjunction with the displacement hypothesis, which asserts that given a person's time is constant, the more time the person spends online, the less time they have available for participating in daily activities offline (such as sleep and physical activities; Nie \& Hillygus, 2002). In sum, one potential explanation for why IA is positively associated with poor sleep quality is that individuals with IA are likely to overuse the Internet before going to bed, leading to bedtime procrastination, which may in turn ultimately result in having a diminished quality of sleep and/or sleep deprivation.

Previous studies have found deficits in executive function among individuals with IA (Luo et al., 2007), which manifested chiefly as diminished inhibitory control ability

Table 2. Testing the pathways of the multiple mediation model

\begin{tabular}{lcccc}
\hline & & & \multicolumn{2}{c}{ Percentile bootstrap 95\% CI } \\
\cline { 3 - 5 } Pathway & $\beta$ & SE & 0.02 & Lower \\
\hline $\begin{array}{l}\text { Internet addiction-Rumination-Poor } \\
\text { sleep quality }\end{array}$ & 0.12 & 0.02 & 0.03 & 0.16 \\
$\begin{array}{l}\text { Internet addiction-Bedtime } \\
\text { procrastination-Poor sleep quality }\end{array}$ & 0.06 & 0.01 & 0.02 & 0.07 \\
$\begin{array}{l}\text { Internet addiction-Rumination- } \\
\quad \text { Bedtime procrastination-Poor sleep } \\
\quad \text { quality }\end{array}$ & 0.05 & 0.03 & 0.18 & 0.28 \\
\begin{tabular}{l} 
Total indirect effect \\
\hline
\end{tabular}
\end{tabular}


(Dong, Lu, Zhou, \& Zhao, 2010). When faced with inhibition tasks, inhibition control among those with IA becomes inefficient, leading to excessive consumption of psychological resources (Dong et al., 2010) and increased ruminative thinking (Ma, Zhao, Wang, Yang, \& Qian, 2013). This in turn produces more negative affect (Lai et al., 2009), consistent with the findings of Constantin et al. (2018), Flett et al. (2016), and Stainton et al. (2000). Further, individuals with high levels of negative affect appear to consider sleeping during set times tedious, with the derived benefits from doing so (i.e., energy and mental health induced by good sleep) being delayed and long-term. Consequently, such individuals are more likely to engage in tasks that are more immediately pleasurable and enjoyable (e.g., watching TV, listening Music) for alleviating current negative affect rather than devoting time to activities absent immediate positive rewards (such as going to sleep on time). Repeated failed self-regulation efforts to alleviate negative affect perpetuate continued delays in bedtime (Exelmans \& Van den Bulck, 2018) and avoidance of healthy self-regulatory behaviors, such as sleeping on time (Sirois, 2016; Song, Su, \& Feng, 2015; Wagner \& Heatherton, 2015). Overall, people with IA typically reveal high levels of rumination and corresponding increased negative affect. In order to effectively manage their current negative affect, individuals with IA are more prone to increase their involvement in recreational activities, which leads to further bedtime delays, ultimately reducing sleep quality.

We also found that IA can reduce sleep quality directly through rumination, consistent with Liu et al. (2017) who showed that rumination mediated the relationship between mobile phone addiction and sleep quality. Pre-sleep arousal includes cognitive and somatic arousal, which are known to be risk factors for sleep problems (Nicassio, Mendlowitz, Fussell, \& Petras, 1985). Our findings, showing increased rumination due to IA, suggest high levels of cognitive and somatic arousal may have been produced as well (Yeh, Wung, \& Lin, 2015), further contributing to difficulties initiating and/or maintaining sound, sustained sleep (Espie, 2007; Guastella \& Moulds, 2007).

The cross-sectional design implemented in this initial, exploratory investigation limits our ability to make strong causal statements about the nature of the relationships between IA and poor sleep quality. Further research, utilizing controlled experimental or longitudinal designs, are needed to more rigorously examine the potential causal links between IA and poor sleep quality and clarify relationships within the mediation model. SEM mediational analyses nonetheless are helpful in the initial stages of research to provide strong hints of causality, thus laying the groundwork for the more extensive, effortful, and expensive longitudinal studies that are needed to support specific claims of causality. Our sample of convenience, nonclinical college students, limits the extent to which we are able to generalize our results to individuals with the most severe forms of IA. Having laid the groundwork, our findings point to a need to replicate and extend this line of research with more diverse samples over extended periods of time.

\section{CONCLUSIONS}

This study revealed that rumination and bedtime procrastination are important mediators in the relationship between IA and poor sleep quality. Specifically, IA by itself was not sufficient for predicting poor sleep quality, but rumination and bedtime procrastination were each found to be significant independent mediators for the relationship between IA and poor sleep quality. Most important, however, was the support found for the sequential mediating effects of rumination and bedtime procrastination between IA and poor sleep quality.

Funding sources: This work was supported by the Hubei Province Education Science Planning Fund [Program No. 2019GA011].

Authors' contribution: ZY, LZ, and NY created and organized the study and collected the data, ZY analyzed the data; ZY, LZ, WM and FA wrote the first draft, analyzed and interpreted the data, NY provided the directions of data analysis. LZ and FA supervised the study and were responsible for all final editing. FA, ZY, LZ, WM and NY critically reviewed the manuscript and provided constructive comments.

Conflict of interest: The authors declare no conflict of interest.

\section{REFERENCES}

Alimoradi, Z., Lin, C. Y., Broström, A., Bülow, P. H., Bajalan, Z., Griffiths, M. D., .. \& \& Pakpour, A. H. (2019). Internet addiction and sleep problems: A systematic review and meta-analysis. Sleep Medicine Reviews, 47, 51-61. https://doi.org/10.1016/j. smrv.2019.06.004

Andreassen, C. S., Griffiths, M. D., Gjertsen, S. R., Krossbakken, E., Kvam, S., \& Pallesen, S. (2013). The relationships between behavioral addictions and the five-factor model of personality. Journal of Behavioral Addictions, 2(2), 90-99. https://doi.org/ 10.1556/JBA.2.2013.003.

Bai, Y., \& Fan, F. (2005). A study on the Internet Dependence of college students: The revising and applying of a measurement. Psychological Development and Education (China), 21(4), 99104.

Bener, A., Yildirim, E., Torun, P., Çatan, F., Bolat, E., Alıc, S., et al. (2019). Internet addiction, fatigue, and sleep problems among adolescent students: A large-scale study. International Journal of Mental Health and Addiction, 17(4), 959-969. https://doi. org/10.1007/s11469-018-9937-1.

Brunborg, G. S., Mentzoni, R. A., Molde, H., Myrseth, H., Skouverøe, K. J. M., Bjorvatn, B., et al. (2011). The relationship between media use in the bedroom, sleep habits and symptoms of insomnia. Journal of Sleep Research, 20(4), 569-575. https:// doi.org/10.1111/j.1365-2869.2011.00913.x. 
Butz, S., \& Stahlberg, D. (2018). Can self-compassion improve sleep quality via reduced rumination? Self and Identity, 17(6), 666686. https://doi.org/10.1080/15298868.2018.1456482.

Buysse, D. J., Reynolds , III, C. F., Monk, T. H., Berman, S. R., \& Kupfer, D. J. (1989). The Pittsburgh sleep quality index: A new instrument for psychiatric practice and research. Psychiatry Research, 28(2), 193-213. https://doi.org/10.1016/01651781(89)90047-4.

Cain, N., \& Gradisar, M. (2010). Electronic media use and sleep in school-aged children and adolescents: A review. Sleep Medicine, 11(8), 735-742. https://doi.org/10.1016/j.sleep.2010.02.006.

Cain, M. K., Zhang, Z., \& Yuan, K. H. (2017). Univariate and multivariate skewness and kurtosis for measuring nonnormality: Prevalence, influence and estimation. Behavior Research Methods, 49(5), 1716-1735. https://doi.org/10.3758/s13428-016-0814-1.

Callegaro, M., \& DiSogra, C. (2008). Computing response metrics for online panels. Public Opinion Quarterly, 72(5), 1008-1032. https://doi.org/10.1093/poq/nfn065.

Çelebioğlu, A., Aytekin Özdemir, A., Küçükoğlu, S., \& Ayran, G. (2020). The effect of Internet addiction on sleep quality in adolescents. Journal of Child and Adolescent Psychiatric Nursing, 33(4), 221-228. https://doi.org/10.1111/jcap.12287.

Chen, Y. L., \& Gau, S. S. F. (2016). Sleep problems and internet addiction among children and adolescents: A longitudinal study. Journal of Sleep Research, 25(4), 458-465. https://doi.org/ 10.1111/jsr.12388.

Chen, I. H., Pakpour, A. H., Leung, H., Potenza, M. N., Su, J. A., Lin, C. Y., et al. (2020). Comparing generalized and specific problematic smartphone/internet use: Longitudinal relationships between smartphone application-based addiction and social media addiction and psychological distress. Journal of Behavioral Addictions, 9(2), 410-419. https://doi.org/10.1556/ 2006.2020.00023.

Christakis, D. A., Moreno, M. M., Jelenchick, L., Myaing, M. T., \& Zhou, C. (2011). Problematic internet usage in US college students: A pilot study. BMC Medicine, 9(1), 77. https://doi.org/ 10.1186/1741-7015-9-77.

Chung, T. W., Sum, S. M., \& Chan, M. W. (2019). Adolescent Internet addiction in Hong Kong: Prevalence, psychosocial correlates, and prevention. Journal of Adolescent Health, 64(6), S34-S43. https://doi.org/10.1016/j.jadohealth.2018.12.016.

Constantin, K., English, M. M., \& Mazmanian, D. (2018). Anxiety, depression, and procrastination among students: Rumination plays a larger mediating role than worry. Journal of RationalEmotive \& Cognitive-Behavior Therapy, 36(1), 15-27. https:// doi.org/10.10 07/s10942-017-0271-5.

Cui, L. J., \& Wang, X. H. (2003). A review on the influence of the Internet on the psychological development of adolescents. Psychological Science (China), 26(3), 501-503.

Davis, R. A. (2001). A cognitive-behavioral model of pathological Internet use. Computers in Human Behavior, 17(2), 187-195. https://doi.org/10.1016/s0747-5632(00)00041-8.

Dong, G., Lu, Q., Zhou, H., \& Zhao, X. (2010). Impulse inhibition in people with Internet addiction disorder: Electrophysiological evidence from a Go/NoGo study. Neuroscience Letters, 485(2), 138-142. https://doi.org/10.1016/j.neulet. 2010.09.002.
Espie, C. A. (2007). Understanding insomnia through cognitive modelling. Sleep Medicine, 8, S3-S8. https://doi.org/10.1016/ S1389-9457(08)70002-9.

Exelmans, L., \& Van den Bulck, J. (2016). Bedtime mobile phone use and sleep in adults. Social Science \& Medicine, 148, 93-101. https://doi.org/10.1016/j.socscimed.2015.11.037.

Exelmans, L., \& Van den Bulck, J. (2017). "Glued to the Tube": The interplay between self-control, evening television viewing, and bedtime procrastination. Communication Research. https://doi. org/10.1177/0093650216686877.

Exelmans, L., \& Van den Bulck, J. (2018). Self-control depletion and sleep duration: The mediating role of television viewing. Psychology \& Health, 33(10), 1251-1268. https://doi.org/10. 1080/08870446.2018.1489048.

Falavigna, A., de Souza Bezerra, M. L., Teles, A. R., Kleber, F. D., Velho, M. C., Steiner, B., et al. (2011). Sleep disorders among undergraduate students in Southern Brazil. Sleep and Breathing, 15(3), 519-524. https://doi.org/10.1007/s11325-010-0396-6.

Feng, J., Zeng, S., Sun, Y., \& Kuroki, K. (2014). A study on the relationship between undergraduate's Internet addiction disorder, online game experience, online time and sleep quality. World Journal of Sleep Medicine (China), 1(4), 193-197.

Flett, A. L., Haghbin, M., \& Pychyl, T. A. (2016). Procrastination and depression from a cognitive perspective: An exploration of the associations among procrastinatory automatic thoughts, rumination, and mindfulness. Journal of Rational-Emotive \& Cognitive-Behavior Therapy, 34(3), 1-18. https://doi.org/10. 1007/s10942-016-0235-1.

Gort, C., Marcusson-Clavertz, D., \& Kuehner, C. (2020). Procrastination, affective state, rumination, and sleep quality: Investigating reciprocal effects with ambulatory assessment. Journal of Rational-Emotive \& Cognitive-Behavior Therapy, 1-28. https:// doi.org/10.1007/s10942-020-00353-4.

Greszki, R., Meyer, M., \& Schoen, H. (2015). Exploring the effects of removing "Too Fast" responses and respondents from Web surveys. Public Opinion Quarterly, 79(2), 471-503. https://doi. org/10.1093/poq/nfu058.

Guastella, A. J., \& Moulds, M. L. (2007). The impact of rumination on sleep quality following a stressful life event. Personality and Individual Differences, 42(6), 1151-1162. https://doi.org/10. 1016/j.paid.2006.04.028.

Han, X., \& Yang, H. (2009). Chinese version of Nolen-Hoeksema ruminative responses scale (RRS) used in 912 college students: Reliability and validity. Chinese Journal of Clinical Psychology, 17(5), 550-551. https://doi.org/10.16128/j.cnki.1005-3611.2009. 05.028.

Jahan, S. M., Hossain, S. R., Sayeed, U. B., Wahab, A., Rahman, T., \& Hossain, A. (2019). Association between internet addiction and sleep quality among students: A cross-sectional study in Bangladesh. Sleep and Biological Rhythms, 17(3), 323-329. https://doi.org/10.1007/s41105-019-00219-y.

Joormann, J., \& Quinn, M. E. (2014). Cognitive processes and emotion regulation in depression. Depression and Anxiety, 31(4), 308-315. https://doi.org/10.1002/da.22264.

Kadzikowska-Wrzosek, R. (2018). Insufficient sleep among adolescents: The role of bedtime procrastination, chronotype and autonomous vs. controlled motivational regulations. Current 
Psychology, 39, 1031-1040. https://doi.org/10.1007/s12144-0189825-7.

Kline, R. B. (2015). Principles and practice of structural equation modeling. Guilford publications.

Kroese, F. M., De Ridder, D. T., Evers, C., \& Adriaanse, M. A. (2014). Bedtime procrastination: Introducing a new area of procrastination. Frontiers in Psychology, 5, 611. https://doi.org/ 10.3389/fpsyg.2014.00611.

Kroese, F. M., Evers, C., Adriaanse, M. A., \& de Ridder, D. T. (2016). Bedtime procrastination: A self-regulation perspective on sleep insufficiency in the general population. Journal of Health Psychology, 21(5), 853-862. https://doi.org/10.1177/ 1359105314540014.

Kuang, X., Cao, Y., \& Dai, X. (2011). Meta-analysis of Internet addiction scales' reliability and validity. Chinese Journal of Clinical Psychology, 19, 192-194. https://doi.org/10.16128/j. cnki.1005-3611.2011.02.040.

Kuss, D. J., \& Griffiths, M. D. (2011). Online social networking and addiction-a review of the psychological literature. International Journal of Environmental Research and Public Health, 8(9), 3528-3552. https://doi.org/10.3390/ijerph8093528.

Lai, S., Han, X., \& Yang, H. (2009). A review of rumination research abroad. Chinese Journal of Applied Psychology, 15(1), 90-96. https://doi.org/10.3969/j.issn.1006-6020.2009.01.015.

Lam, L. T. (2014). Internet gaming addiction, problematic use of the internet, and sleep problems: A systematic review. Current Psychiatry Reports, 16(4), 444. https://doi.org/10.1007/s11920014-0444-1.

Liu, X., Tang, M., \& Hu, L. (1996). Reliability and validity of the Pittsburgh sleep quality index. Chinese Journal of Psychiatry, 29, 103-107. https://doi.org/10.1007/BF02951625.

Liu, Q. Q., Zhou, Z. K., Yang, X. J., Kong, F. C., Niu, G. F., \& Fan, C. Y. (2017). Mobile phone addiction and sleep quality among Chinese adolescents: A moderated mediation model. Computers in Human Behavior, 72, 108-114. https://d oi.org/10.1016/j.chb. 2017.02.042.

Li, Y., Zhong, B., Liu, X., Zhang, Y., Zhu, J., \& Hao, W. (2012). Reliability and validity of the Chinese version of self-rating Young's diagnostic questionnaire of internet addiction: A preliminary study. Chinese Journal of Drug Dependence, 21(5), 390-394.

Lu, J. G., Liu, X. L., Liao, H., \& Wang, L. (2020). Disentangling stereotypes from social reality: Astrological stereotypes and discrimination in China. Journal of Personality and Social Psychology. Advance online publication. https://doi.org/10. 1037/pspi0000237.

Luo, Q., Meng, Q., Fu, Y., Du, L., Du, X., Yang, H., et al. (2007). A case-control study of cognitive function of internet addicts. Chinese Mental Health Journal, (4), 237-239. https://doi.org/10. 3321/j.issn:1000-6729.2007.04.009.

Mardia, K. V. (1970). Measures of multivariate skewness and kurtosis with applications. Biometrika, 57(3), 519-530. https:// doi.org/10.1093/biomet/57.3.519.

Ma, X., Zhao, Q., Wang, M., Yang, Y., \& Qian, M. (2013). Inhibition deficits for emotional words in high ruminators. Chinese Mental Health Journal, 27(12), 918-923. https://doi.org/10. 3969/j.issn.1000-6729.2013.12.007.

Nauts, S., Kamphorst, B. A., Stut, W., De Ridder, D. T., \& Anderson, J. H. (2018). The explanations people give for going to bed late: A qualitative study of the varieties of bedtime procrastination. Behavioral Sleep Medicine, 17(6), 753-762. https://doi.org/10.1080/15402002.2018.1491850.

Nicassio, P. M., Mendlowitz, D. R., Fussell, J. J., \& Petras, L. (1985). The phenomenology of the pre-sleep state: The development of the pre-sleep arousal scale. Behaviour Research and Therapy, 23, 263-271. https://doi.org/10.1016/ 0005-7967(85)90004-X.

Nie, N. H, \& Hillygus, D. S. (2002). Where does internet time come from? A reconnaissance. IT \& Society, 1(2), 1-20.

Niu, G. F., Sun, X. J., Subrahmanyam, K., Kong, F. C., Tian, Y., \& Zhou, Z. K. (2016). Cue-induced craving for Internet among Internet addicts. Addictive Behaviors, 62, 1-5. https://doi.org/ 10.1016/j.addbeh.2016.06.012.

Nolen-Hoeksema S. (1991). Responses to depression and their effects on the duration of depressive episodes. Journal of Abnormal Psychology, 100(4),569-582. https://doi.org/10.1037// 0021-843X.100.4.569.

Nolen-Hoeksema, S., \& Morrow, J. (1991). A prospective study of depression and posttraumatic stress symptoms after a natural disaster: The 1989 loma Prieta Earthquake. Journal of Personality and Social Psychology, 61(1), 115-121. https://doi.org/10. 1037/0022-3514.61.1.115.

Pawlikowski, M., \& Brand, M. (2011). Excessive Internet gaming and decision making: Do excessive World of Warcraft players have problems in decision making under risky conditions? Psychiatry Research, 188(3), 428-433. https://doi.org/10.1016/j. psychres.2011.05.017.

Peng, L., \& Xie, T. (2016). Making similarity versus difference comparison affects perceptions after bicultural exposure and consumer reactions to culturally mixed products. Journal of Cross-Cultural Psychology, 47, 1380-1394. http://dx.doi.org/10. 1177/0022022116668409.

Przepiórka, A., Błachnio, A., \& Siu, N. Y. F. (2019). The relationships between self-efficacy, self-control, chronotype, procrastination and sleep problems in young adults. Chronobiology International, 36(8), 1025-1035. https://doi.org/10.1080/ 07420528.2019.1607370.

Rebetez, M. M. L., Rochat, L., Barsics, C., \& Van der Linden, M. (2018). Procrastination as a self-regulation failure: The role of impulsivity and intrusive thoughts. Psychological Reports, 121(1), 26-41. https ://doi.org/10.1177/0033294117 720695.

Shek, D. T., \& Yu, L. (2016). Adolescent internet addiction in Hong Kong: Prevalence, change, and correlates. Journal of Pediatric and Adolescent Gynecology, 29(1), S22-S30. https://doi.org/10. 1016/j.jpag.2015.10.005.

Sirois, F. M. (2007). "I'll look after my health, later": A replication and extension of the procrastination-health model with community-dwelling adults. Personality and Individual Differences, 43(1):15-26. https://doi.org/10.1016/j.paid.2006.11.003.

Sirois, F. M. (2016). Perfectionism and health behaviors: A selfregulation resource perspective. In F. Sirois \& D. Molnar. (Eds). Perfectionism, health, and well-being (pp. 45-67). Cham: Springer.

Sirois, F. M., Nauts, S., \& Molnar, D. S. (2019). Self-compassion and bedtime procrastination: An emotion regulation perspective. Mindfulness, 10(3), 434-445. https://doi.org/10.1007/s12671018-0983-3. 
Song, M., Su, T., \& Feng, T. (2015). The model of procrastination on time orientation. Advances in Psychological Science, 23(7), 1216-1225. https://doi.org/10.3724/SP.J.1042.2015.01216.

Stainton, M., Lay, C. H., \& Flett, G. L. (2000). Trait procrastinators and behavior/trait-specific cognitions. Journal of Social Behavior and Personality, 15(5), 297-312. https://doi.org/10. 1080/00926230152035859.

Steel, P., \& König, C. J. (2006). Integrating theories of motivation. Academy of Management Review, 31(4), 889-913. https://doi. org/10.5465/AMR.2006.22527462.

Straub, D. W. (1989). Validating instruments in MIS research. MIS Quarterly, 13(2), 147-169. https://doi.org/10.2307/248922.

Suganuma, N., Kikuchi, T., Yanagi, K., Yamamura, S., Morishima, H., Adachi, H., .. \& \& Takeda, M. (2007). Using electronic media before sleep can curtail sleep time and result in selfperceived insufficient sleep. Sleep and Biological Rhythms, 5(3), 204-214. https://doi.org/10.1111/j.1479-8425.2007. 00276.x.

Tofighi, D. (2020). Bootstrap model-based constrained optimization tests of indirect effects. Frontiers in Psychology, 10, 2989. https://doi.org/10.3389/fpsyg.2019.02989.

Tofighi, D., \& Kelley, K. (2020). Indirect effects in sequential mediation models: Evaluating methods for hypothesis testing and confidence interval formation. Multivariate Behavioral Research, 55(2), 188-210. https://doi.org/10.1080/00273171. 2019.1618545.

Treynor, W., Gonzalez, R., \& Nolen-Hoeksema, S. (2003). Rumination reconsidered: A psychometric analysis. Cognitive Therapy and Research, 27, 247-259. https://doi.org/10.1023/A: 1023910315561.

Vadher, S. B., Panchal, B. N., Vala, A. U., Ratnani, I. J., Vasava, K. J., Desai, R. S., et al. (2019). Predictors of problematic Internet use in school going adolescents of Bhavnagar, India. International Journal of Social Psychiatry, 65(2), 151-157. https://doi. org/10.1177/0020764019827985.

Wagner, D. D., \& Heatherton, T. F. (2015). Self-regulation and its failure: The seven deadly threats to self-regulation. InAPA handbook of personality and social psychology, Volume 1: Attitudes and social cognition. (pp. 805-842). American Psychological Association.

Wang, W. (2018). An overview of the mechanism of social networking addiction and its negative consequences. Advances in Psychology, 8(8), 1057-1063. https://doi.org/10.1 2677/AP. 2018.88127.

Wang, P., Chen, K., Yang, S., \& Lin, P. (2019). Relationship of sleep quality, smartphone dependence, and health-related behaviors in female junior college students. PloS One, 14(4), e0214769. https://doi.org/10.1371/journal.pone.0214769.

Wang, Q., Liu, Y., Wang, B., An, Y., Wang, H., Zhang, Y., et al. (2020). Problematic internet use and subjective sleep quality among college students in China: Results from a pilot study. Journal of American College Health. https://doi.org/10.1080/ 07448481.2020 .1756831$.

Wood, R. T., Griffiths, M. D., \& Parke, A. (2007). Experiences of time loss among videogame players: An empirical study. CyberPsychology \& Behavior, 10(1), 38-44. http://dx.doi.org/10. 1089/cpb.2006.9994.

Yeh, Z. T., Wung, S. K., \& Lin, C. M. (2015). Pre-sleep arousal as a mediator of relationships among worry, rumination, and sleep quality. International Journal of Cognitive Therapy, 8(1), 21-34. https://doi.org/10.1521/ijct.2015.8.1.21.

Younes, F., Halawi, G., Jabbour, H., El Osta, N., Karam, L., Hajj, A., et al. (2016). Internet addiction and relationships with insomnia, anxiety, depression, stress and self-esteem in university students: A cross-sectional designed study. PloS One, 11(9), e0161126. https://doi.org/10.1371/journal.pone. 0161126.

Young, K. S. (1998). Internet addiction: The emergence of a new clinical disorder. CyberPsychology \& Behavior, 1(3), 237-244. https://doi.org/10.1089/cpb.1998.1.237.

Zhang, M. W., Tran, B. X., Hinh, N. D., Nguyen, H. L. T., Tho, T. D., Latkin, C., et al. (2017). Internet addiction and sleep quality among Vietnamese youths. Asian Journal of Psychiatry, 28, 1520. https://doi.org/10.1016/j.ajp.2017.03.025. 